\title{
Síncope 2018: ¿qué hay de nuevo en las guías europeas?
}

\author{
Syncope 2018: what is new in the european guidelines? \\ Dr. Gabriel Vanerio
}

Una adolescente de 16 años se desmaya luego de bailar, una mujer de 67 años consulta por episodios de pérdida de conocimiento y traumatismos severos en los últimos meses. Un hombre de 75 años con cardiopatía isquémica conocida consulta por palpitaciones rápidas y síncope. Estos tres ejemplos son relativamente frecuentes en la práctica diaria. $\mathrm{Si}$ bien a primera vista el diagnóstico y tratamiento parecen complejos, las nuevas guías de síncope 2018 de la Sociedad Europea de Cardiología ofrecen una rápida orientación, algoritmos diagnósticos y un tratamiento para cada caso ${ }^{(1,2)}$.

Las guías 2018 deben ser leídas en detalle, este editorial-comentario no reemplaza su lectura, son 69 páginas que aportan información muy importante, revisada y ordenada. Resumen las recomendaciones diagnósticas y terapéuticas, agregando la evidencia y el conocimiento adquirido en los últimos nueve años ${ }^{(1,2)}$. Se trata de un documento multidisciplinario (neurología, cardiología, medicina interna, medicina familiar, urgencias, enfermería), donde, además, se subraya la importancia del trabajo en equipo.

\section{Diagnóstico clínico (tabla 1)}

Durante la evaluación clínica inicial se puede definir la causa de síncope en la mayoría de los casos. Es fundamental entender y adherir rigurosamente a las definiciones de síncope vasovagal o síncope reflejo, tanto sea situacional o debido a hipotensión ortostática. En estos casos el mecanismo puede ser muy evidente o altamente probable, independientemente de la presencia de algún otro hallazgo anormal. En sujetos jóvenes sanos con síncope de causa no aclarada o sin explicación y sin otras alteraciones (por ejemplo, sin enfermedad cardíaca o antecedentes familiares de muerte súbita, sin síncope en decúbito o durante ejercicio o durante el sueño), sin claros desencadenantes y con electrocardiograma normal, las chances de síncope cardíaco maligno son muy bajas. Las tasas de muerte súbita en esta población menor a 35 años son de 1 a 3 por 100.000 personas.

Los episodios sincopales de bajo riesgo tienen los pródromos característicos del síncope reflejo (vasovagal): mareos, sensación de calor, sudoración, náuseas y vómitos. Ocurren luego de un evento súbito inesperado, como puede ser dolor, visión de algo desagradable, o simplemente escuchar algo repulsivo, incluso frente a un olor repugnante; luego de estación prolongada de pie, en lugares cerrados o calurosos, con mucha gente; durante ingestas o posprandial, y también pueden ser desencadenados por tos, defecación y micción.

Todas las formas de síncope, pero sobre todo el síncope reflejo y la hipotensión ortostática tienen una presentación más severa cuando hay otros factores asociados, como uso de medicación vasodilatadora, alcohol, depleción de volumen, hemorragia, escaso consumo de líquidos, diarrea, vómitos y otros factores ambientales.

Hay dos mecanismos básicos en el síncope reflejo: a) la vasodepresión que indica ausencia de vasoconstricción simpática resultando en hipotensión;

Servicio de Electrofisiología, Departamento de Cardiología. Hospital Británico.

Servicio de Electrofisiología y Arritmias del CASMU.

Correspondencia: Dr. Gabriel Vanerio. Hospital Británico, UCI, Piso 2. Av. Italia 2420.

Correo electrónico: gabriel.vanerio@yahoo.com

Recibido Jul 23, 2018; aceptado Set 1, 2018. 


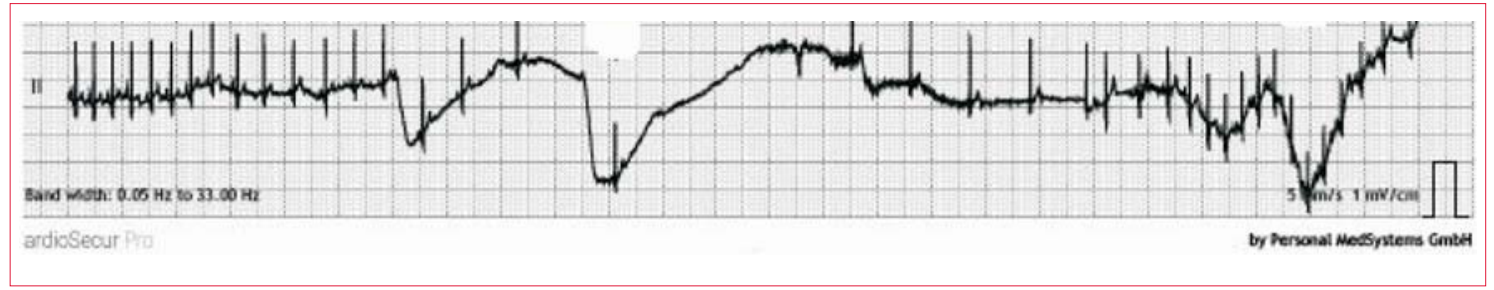

Figura 1. Síncope reflejo vasovagal con componente cardioinhibitorio. Electrocardiograma mostrando la derivación DII a $5 \mathrm{~mm} / \mathrm{s}$ durante una prueba de inclinación de un paciente de 16 años, con historia de episodios sincopales luego de dolor intenso, el último postraumatismo de rodilla derecha. Se puede observar cómo la frecuencia cardíaca se va enlenteciendo progresivamente hasta que desarrolla asistolia por casi $9 \mathrm{~s}$. Luego de la misma, el paciente se recupera completamente.

\section{Tabla 1. Puntos clave a recordar}

El síncope se define como una pérdida transitoria de la conciencia debido a hipoperfusión cerebral, caracterizada por un inicio rápido, corta duración y recuperación completa espontánea.

El síncope no es un diagnóstico, es un síntoma.

La consulta en emergencia es extremadamente frecuente, alcanzando 3\% de todas las consultas.

En la evaluación inicial los clínicos deben responder cinco preguntas fundamentales:

1. ¿Fue una pérdida transitoria de la conciencia?

2. En caso de ser una pérdida transitoria de la conciencia, ies de origen sincopal o no?

3. En caso de presunto síncope, iexiste un diagnóstico etiológico claro?

4. ¿Existen pruebas que sugieran alto riesgo de un evento cardiovascular o muerte? ¿Existe una causa subyacente que pueda ser identificada?

5. Si la causa es incierta, ¿cuál es el riesgo de un evento grave? ¿Se debe hospitalizar al paciente?

Todos los pacientes deben ser muy bien interrogados, es fundamental obtener una historia completa, detallada (detectivesca) y si es posible que incluya testigos. El examen físico debe contar además con medición de la presión arterial en posición de pie, y un electrocardiograma estándar (ECG 12 derivaciones).

La presencia de movimientos anormales descritos por testigos es una observación frecuente. Los movimientos anormales son muy típicos, característicos, breves, con movimiento de la cabeza y de los miembros superiores, a manera de clonias (sacudidas). Ocurren en la fase de recuperación a diferencia de los movimientos anormales característicos de la crisis epiléptica (ver video asociado).

b) la cardioinhibición cuando hay predominio de bradicardia o asistolia (figura 1).

El patrón hemodinámico, sea cardioinhibitorio o vasodepresor, es independiente del disparador del reflejo. Ambas formas pueden estar presentes tanto en el síncope miccional como en el síncope vasovagal postural, por ejemplo.

Las formas no clásicas de síncope reflejo involucran a un grupo heterogéneo de pacientes. El término se usa para describir un síncope que ocurre sin claros desencadenantes, por lo que la presentación es atípica. El diagnóstico de este tipo de síncope reflejo es posible cuando se excluyen otras causas.

Existen elementos clínicos y electrocardiográficos que permiten sospechar una etiología cardíaca, estructural o eléctrica, potencialmente de importancia pronóstica, que se resumen en la tabla 2.

\section{Estudios diagnósticos}

\section{a) Estudios de tolerancia ortostática}

Existen tres métodos para evaluar la respuesta al cambio de postura de acostado a parado:

1. Test parado activo.

2. Prueba de inclinación.

3. Monitoreo ambulatorio de presión arterial.

Prueba de inclinación

La prueba de inclinación (PI) se debe considerar como un estudio que desenmascara la tendencia a la hipotensión más que un estudio diagnóstico para síncope vasovagal. Esta susceptibilidad a la hipotensión explica el síncope independientemente de la etiología y el mecanismo. Por ejemplo, en un síncope arrítmico provocado por taquicardia, el mecanismo es la combinación del comienzo de la arritmia en sí misma y la susceptibilidad a la hipotensión ${ }^{(1,2)}$.

Una respuesta positiva cardioinhibitoria durante la PI pronostica con alta probabilidad la presencia de síncope espontáneo asistólico. Este hallazgo es relevante para considerar la terapia con marcapasos.

Durante la PI se recomienda grabar en video el episodio sincopal, con la finalidad no solo de documentar sino de poder analizar el tipo de movimientos, can- 
tidad y duración. Hay ocasiones en que es complejo diferenciar entre crisis convulsivas y síncope con movimientos anormales. La mayoría de los neurólogos opta por realizar, en casos seleccionados, monitoreo electroencefalográfico simultáneo. Su uso podría ayudar en la elaboración diagnóstica para diferenciar entre síncope, epilepsia o pseudosíncope.

El uso extendido de smartphones permite a la mayoría de las personas registrar videos. El único inconveniente es la rapidez para iniciar la grabación, ya que la mayoría de los episodios son breves (<30 segundos) y no dan tiempo. El hecho de que siempre son muy desagradables genera enorme angustia y la mayoría de los testigos optarán por ayudar a la persona durante el episodio en lugar de grabar un video.

\section{Pseudosíncope psicógeno}

Es una condición relativamente común (aproximadamente 5\%-20\% de las consultas). Es más frecuente en mujeres jóvenes y se caracteriza por presentar episodios frecuentes (a veces varios en el mismo día). El interrogatorio es fundamental, debiendo incluir: frecuencia de los episodios, circunstancias e inicio.

La PI es muy útil en el pseudosíncope psicógeno, ya que durante la misma el patrón hemodinámico permanece normal (sin alteraciones), pero el paciente parece perder el conocimiento. Este tipo de síncope a menudo se asocia con abuso sexual.

\section{Monitoreo ambulatorio de la presión arterial}

El monitoreo ambulatorio de la presión arterial no ha demostrado demasiada utilidad en estos pacientes en comparación con las medidas rutinarias de PA en el consultorio.

\section{Estudios de función autonómica básicos}

Hiperventilación o respiración profunda. Bajo condiciones fisiológicas la frecuencia cardíaca aumenta durante la inspiración profunda y disminuye durante la espiración. Se puede usar el índice espiración/inspiración, cuyo valor normal es mayor a 15 pm en individuos sanos de más de 50 años. La ausencia o la abolición de esta variación es sugestiva de disfunción parasimpática.

\section{b) Otros estudios}

\section{Masaje del seno carotídeo}

El masaje del seno carotídeo se debe realizar en pacientes mayores de 40 años con síncope compatible con un mecanismo reflejo. El desarrollo de una pausa de más de 3 segundos o una caída de la PA sistólica de más de $50 \mathrm{mmHg}$ se conoce como hipersensibilidad del seno carotídeo. La hipersensibilidad al masaje del seno carotídeo es excepcional en pacientes menores
Tabla 2. Características clínicas y electrocardiográficas que sugieren síncope cardíaco o potencialmente maligno.

Características clínicas

- Síncope de esfuerzo o en posición supina

- Palpitaciones de inicio súbito seguidas de síncope

- Antecedentes familiares de muerte súbita en $<35$ años

- Presencia de cardiopatía estructural o enfermedad coronaria

Características electrocardiográficas

- Bradicardia sinusal o fibrilación auricular con alta tasa de bloqueo AV (40-50 lpm) en ausencia de medicación que la justifique

- Bloqueo bifascicular (bloqueo de rama izquierda o bloqueo de rama derecha con hemibloqueo anterior o posterior izquierdos)

- Otros trastornos de la conducción intraventricular con QRS > $120 \mathrm{~ms}$

- Bloqueo AV de segundo grado Mobitz I con intervalo PR muy prolongado (280 $\mathrm{ms})$

- Taquicardia ventricular no sostenida

- QT largo o corto

- Preexcitación ventricular

- Patrón de Brugada tipo I

- Repolarización precoz con elevación de ST

- Ondas T negativas en precordiales derechas y onda épsilon (probable displasia del ventrículo derecho)

- Hipertrofia ventricular izquierda que sugiere miocardiopatía hipertrófica

Síncope maligno, alto riesgo de muerte súbita

- Bradicardia sinusal $<40$ lpm persistente o pausas $>3$ segundos en paciente despierto no entrenado

- Bloqueo AV de segundo grado Mobitz II o bloqueo $\mathrm{AV}$ de tercer grado

- Bloqueo de rama alternante

- Taquicardia ventricular o supraventricular

- Episodios de taquicardia ventricular polimorfa no sostenida

- Disfunción de marcapaso o cardiodesfibrilador con pausas

- Relacionado a isquemia

- Relacionado a alteraciones cardiopulmonares estructurales: mixoma auricular

AV: aurículoventricular; lpm: latidos por minuto 


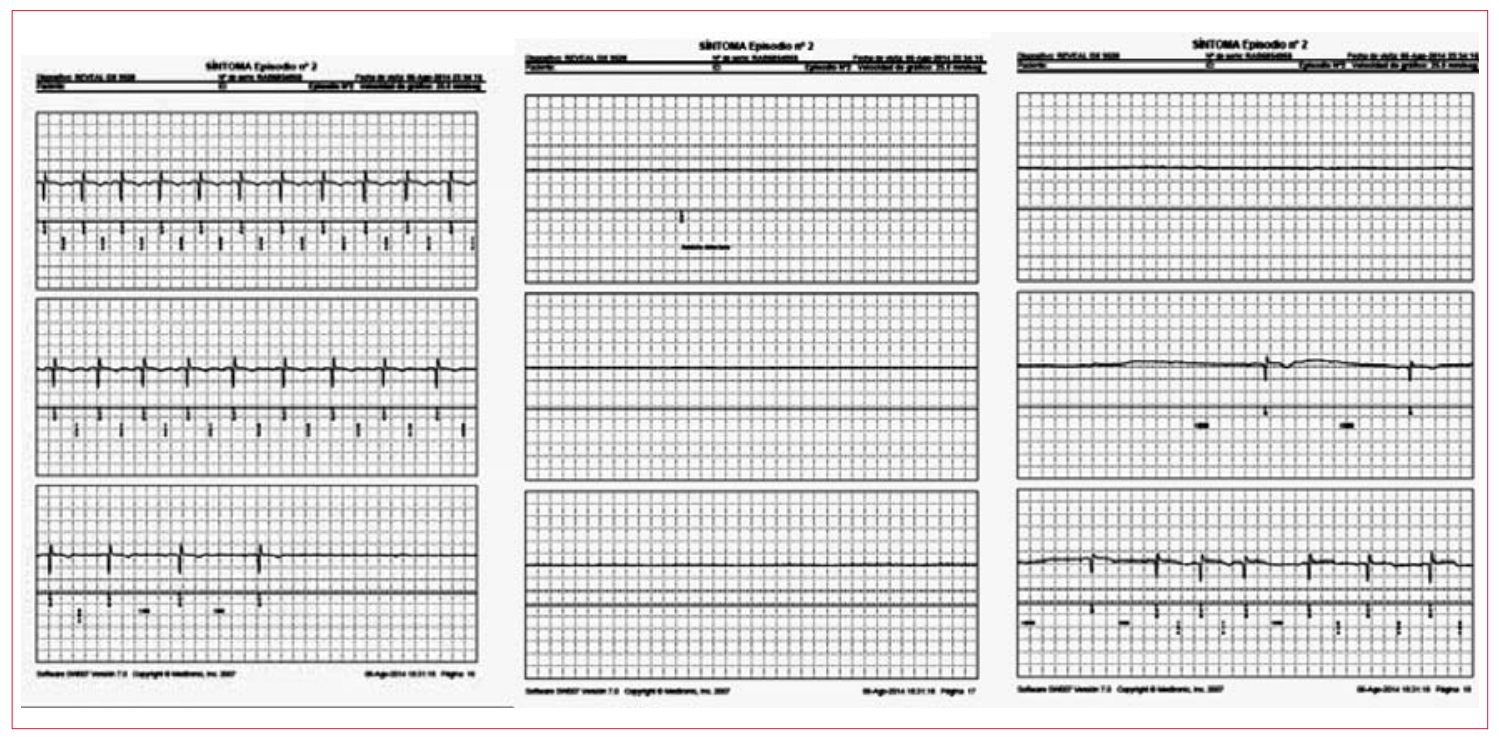

Figura 2. Registro de eventos implantable del caso número 2. Es un trazado continuo. Se observa cómo desarrolla una asistolia de 36 segundos. Luego de alguna pausa secundaria, se recupera completamente.

Tabla 3. Indicaciones de registradores de eventos implantables.

Clase

En pacientes con epilepsia sospechada pero no confirmada

En pacientes con caídas no explicadas IIa

En pacientes con cardiomiopatía primaria o IIa canalopatías congénitas arritmogénicas

Bajo riesgo de muerte súbita como alternativa al implante de un desfibrilador

Como alternativa antes de indicar el desfibrilador: en casos seleccionados

de 40 años. Existe consenso entre la mayoría de los expertos en la definición del síndrome de seno carotídeo hipersensible: debe reproducir los mismos síntomas clínicos y debe ocurrir en sujetos con síncope de causa no aclarada, con características sugestivas de síncope reflejo. La correlación entre el masaje del seno carotídeo y eventos espontáneos ha sido evaluada indirectamente por la eficacia de la estimulación cardíaca. Aún queda por investigar y es probable que en un futuro cercano podamos estimar mejor la utilidad de esta técnica diagnóstica.

\section{Monitor en la emergencia}

Se recomienda el uso de un monitor con capacidad de registro y memoria (cama o por telemetría) en pacientes de riesgo elevado cuando hay sospecha de síncope arrítmico.

\section{Ecocardiograma Doppler}

Cuando existe enfermedad cardíaca conocida previa o sospecha de cardiopatía estructural o síncope secundario a causa cardiovascular.

\section{Monitoreo electrocardiográfico prolongado}

Se debe realizar en pacientes con síncope inexplicado, severo y recurrente con características clínicas o electrocardiográficas que sugieran síncope arrítmico (sin desencadenantes) y con alta probabilidad de recurrencia en un tiempo relativamente prolongado (semanas o meses).

\section{Registradores de eventos implantables}

Su indicación más importante es en el síncope esporádico sin causa evidente (tabla 3 ). En las guías se sugiere ampliar su uso dado que triplican la capacidad diagnóstica cuando se la compara con una estrategia convencional, además, son costo-efectivos. El mensaje más importante es su uso precoz en las etapas diagnósticas (figura 2). Desafortunadamente en nuestro país estos dispositivos no se encuentran cubiertos por el sistema de salud. Debemos bregar por su incorporación a la brevedad dada su enorme capacidad diagnóstica.

\section{Estudio electrofisiológico}

Se debe realizar en los pacientes con síncope no explicado más cardiopatía estructural, o bloqueo de rama o trastorno de la conducción intraventricular (sospecha de bloqueo auriculoventricular [AV] de alto grado) y taquicardia incierta. 
Tabla 4. Estratificación de riesgo.

\section{Criterios de severidad}

- Probable origen isquémico

- Síncope durante el ejercicio o en posición supina

- Palpitaciones de inicio brusco con síncope

- Antecedente de enfermedad cardíaca estructural

- Otros hallazgos en la exploración física (hipotensión y bradicardia persistentes sin explicación, hemorragia gastrointestinal)

- Cambios electrocardiográficos que sugieran enfermedad cardíaca estructural o eléctrica primaria

\section{Ergometría o prueba de esfuerzo}

En pacientes que experimenten síncope durante o muy poco después del esfuerzo.

\section{3) Estudios sin rendimiento diagnóstico}

a) Tomografía de cráneo si el paciente no tuvo traumatismo encéfalo-craneano.

b) Eco Doppler de vasos del cuello. En pacientes menores a 50 años (incluso en sujetos mayores la oclusión carotídea casi nunca se presenta como un cuadro sincopal).

c) El Holter de 24 horas tiene un pobre rendimiento diagnóstico en la enorme mayoría de los casos.

\section{4) Tratamiento en la emergencia}

El manejo del paciente con síncope en el departamento de emergencia exige un equipo multidisciplinario que involucra médicos internistas, cardiólogos, de familia, generalistas, emergencistas y neurólogos. Las guías 2018 establecen un protocolo específico para cada paciente.

El departamento de emergencia de cada institución debe entrenar a su personal para estratificar el riesgo y en consecuencia evaluar si los pacientes deben ser ingresados o no. Los criterios de severidad y la conducta recomendada se muestran en las tablas 4 y 5 .

El médico en la emergencia debe tratar de establecer el diagnóstico, única forma de definir una conducta y una terapéutica adecuadas. El $50 \%$ de las pérdidas de conocimiento tienen una causa obvia. En el otro $50 \%$ se sugiere utilizar un algoritmo donde se categoriza al paciente en bajo y alto riesgo. Los pacientes de bajo riesgo pueden ser dados de alta y se les debe entregar un instructivo con recomendaciones para prevenir nuevos episodios sincopales.

\begin{tabular}{lcc}
\hline Tabla 5. Estratificación de riesgo & \\
\hline Conducta & \multicolumn{1}{|c}{ Recomendación } & $\begin{array}{c}\text { Indicación } \\
\text { clase }\end{array}$ \\
\hline Paciente de bajo riesgo & $\begin{array}{l}\text { Alta del } \\
\text { departamento de } \\
\text { emergencia }\end{array}$ & I \\
$\begin{array}{l}\text { Pacientes de alto } \\
\text { riesgo }\end{array}$ & $\begin{array}{l}\text { Manejo rápido en el } \\
\text { departamento de } \\
\text { emergencia o unidad } \\
\text { de síncope }\end{array}$ & I \\
\hline $\begin{array}{l}\text { Paciente de riesgo } \\
\text { dudoso o de riesgo } \\
\text { intermedio }\end{array}$ & $\begin{array}{l}\text { Observación en la } \\
\text { emergencia o unidad } \\
\text { de síncope en lugar de } \\
\text { ser hospitalizado }\end{array}$ & I \\
\hline & \\
\hline
\end{tabular}

El objetivo es reducir la tasa de admisiones hospitalarias utilizando unidades de observación por 6-24 horas o de síncope y referir al paciente a un especialista en síncope fuera de la emergencia lo antes posible.

\section{5) Manejo en el consultorio}

Aunque las guías se basan en la mejor evidencia científica disponible, el tratamiento se debe adaptar siempre a la necesidad de cada paciente individual.

El algoritmo para el síncope reflejo vasovagal no toma solo en cuenta la presentación inicial, sino también la edad del paciente. La indicación de marcapasos en pacientes con síncope a forma cardioinhibitoria tiene en cuenta la presentación clínica y el resultado de la PI.

A todos los pacientes con síncope reflejo se les debe explicar en detalle el diagnóstico, el riesgo de recurrencia y dar consejos sobre cómo evitar desencadenantes y situaciones peligrosas. Es muy importante dar tranquilidad luego de establecer el diagnóstico. Estas medidas son la piedra angular del tratamiento y tienen un alto impacto en la reducción de recurrencias.

En aquellos pacientes con formas severas de síncope reflejo se deberá seleccionar uno o más de los siguientes tratamientos específicos adicionales según las características clínicas:

- Midodrina o fludrocortisona en pacientes jóvenes con fenotipo hipotensivo.

- Maniobras de contrapresión (incluido entrenamiento en inclinación o tilt-training si es necesario) para pacientes jóvenes con pródromos.

- Uso de registradores de eventos implantables en pacientes seleccionados sin o con pocos pródromos.

- Discontinuar o reducir la terapia antihipertensiva con el objetivo de alcanzar una PA sistólica de aproximadamente $140 \mathrm{mmHg}$ en hipertensos añosos. 
- Implante de marcapasos en pacientes (mayores de 50 años) con forma cardioinhibitoria predominante.

Cuando el diagnóstico es hipotensión ortostática por falla autonómica, uno o más de los siguientes tratamientos específicos adicionales pueden ser seleccionados de acuerdo con la severidad clínica:

- Hidratación adecuada y consumo de sal.

- Discontinuación/reducción de la terapia antihipertensiva.

- Maniobras de contrapresión.

- Compresores abdominales o medias de descanso, o ambos.

- Tacos de madera en la cabecera de la cama $(10-15 \mathrm{~cm})$ para elevar la cabeza y mantenerla elevada durante el sueño o mientras el paciente está acostado.

- Midodrina o fludrocortisona.

\section{Caso 1}

Una paciente de 17 años consulta en emergencia por pérdida de conocimiento. $\mathrm{Al}$ interrogatorio refiere pérdida de conocimiento luego de estar bailando durante dos horas, en el momento estaba parada, en lugar cerrado, caluroso. Había tomado alcohol. Refiere, segundos antes, mareos, visión borrosa ("veo todo negro"), le avisa a una amiga, quien la nota muy pálida y la lleva al baño, donde presenta pérdida de conocimiento con caída al suelo y movimientos anormales. Sufre herida en región superciliar, se recupera rápidamente, la levantan y llevan afuera del local, donde parada, vuelve a presentar pérdida de conocimiento, la acuestan y luego de unos minutos se recupera completamente. Al examen, lúcida, sudorosa, presión arterial 90/60 mmHg y una frecuencia cardíaca de 90 por minuto. Sin otras alteraciones en el examen, herida superciliar derecha. El electrocardiograma es normal, ritmo sinusal de 90 pm.

Claves: la historia es típica y la más frecuente en pacientes jóvenes. Nos brinda información relevante. Los testigos son extremadamente útiles. Factores desencadenantes: actividad física, pobre hidratación, calor, lugar cerrado, estación prolongada de pie y consumo de alcohol, todos factores que provocan hipovolemia. La estratificación de riesgo sugiere que es una paciente de bajo riesgo.

Diagnóstico: síncope reflejo vasovagal neuromediado.

Se deben hacer recomendaciones sobre todo higiénico-dietéticas, como aumentar la ingesta de líquidos, en particular antes de ir a bailar o de reali- zar otro tipo de ejercicio. El pronóstico es excelente si sigue las indicaciones. No es necesario hacer otros estudios cardiológicos, por ejemplo Holter o ecocardiograma, ni consultar neurólogo.

\section{Caso 2}

Mujer de 69 años, es referida a policlínica de Cardiología por episodios de pérdida de conocimiento. Vive sola y tiene antecedentes personales de hipertensión arterial tratada con inhibidores de la enzima convertidora de angiotensina. El examen cardiovascular es normal. La historia nos revela que los episodios son breves, de inicio brusco y esporádicos, uno cada dos a tres meses, no sabemos cuánto duran y ocurren en varias posiciones, acostada, sentada o parada. No hay desencadenantes ni testigos. El electrocardiograma es normal y no tenemos otra orientación.

Claves: síncopes esporádicos sin pródromos, en diferentes posiciones. No disponemos de más información.

Las guías sugieren utilizar un registro de eventos implantable. En este caso, luego de tres meses, presentó otro episodio donde se registra un paro sinusal (figura 2) de varios segundos. Se implantó un marcapasos definitivo. Diagnóstico final: enfermedad del nódulo sinusal con paro sinusal prolongado

\section{Caso 3}

Paciente de 75 años con antecedentes personales de hipertensión, diabetes, e infarto de miocardio hace 15 años. Consulta por episodios de pérdida de conciencia frecuentes, sin pródromos. El electrocardiograma muestra secuela de cara anterior y hemibloqueo anterior izquierdo más bloqueo de rama derecha. El ecocardiograma en la emergencia mostró una fracción de eyección disminuida de $30 \%$ con alteraciones de la motilidad segmentaria.

Es un paciente de alto riesgo, con electrocardiograma anormal y además con deterioro de la función ventricular izquierda. El paciente debe ingresar y se indica estudio electrofisiológico. Es muy probable que la etiología sea una taquicardia ventricular y que se beneficie del implante de un cardiodesfibrilador. No requiere de otros estudios adicionales en este momento.

Claves: síncopes sin pródromos. Cardiopatía estructural, cicatriz, deterioro de la fracción de eyec- 
Tabla 6. Principales diferencias con respecto a las guías 2009.

\begin{tabular}{lcc}
\hline & 2009 & 2018 \\
\hline $\begin{array}{l}\text { 1. Las contraindicaciones al masaje del seno carotídeo fueron retiradas } \\
\text { 2. La indicación de PI }\end{array}$ & I & IIa \\
3. La indicación de PI con propósitos educativos & IIa & IIb \\
4. PI como criterio diagnóstico & IIa \\
$\begin{array}{l}\text { 5. Se recomiendan los registros de video para eventos espontáneos como durante } \\
\text { la PI }\end{array}$ & IIa \\
6. PI para evaluar terapia fue retirada &
\end{tabular}

7. Indicación de Holter

I

IIa

8. El monitoreo electrocardiográfico en el paciente con síncope y arritmias asintomáticas fue retirado

9. El test de adenosina trifosfato fue retirado

10. En el estudio electrofisiológico: tiempo de recuperación del nodo sinusal

11. En el estudio electrofisiológico, el intervalo HV $>70 \mathrm{~ms}$ como guía para indicación de marcapasos

12. Implante de marcapaso empírico en el bloqueo bifascicular

IIa $\quad$ IIb

13. Maniobras físicas de contrapresión (cruzamiento de miembros inferiores). Terapia para síncope reflejo

14. Compresores abdominales para el tratamiento de la hipotensión ortostática

15. Uso de fármacos antiarrítmicos en paciente con síncope y taquicardia ventricular/supraventricular (opinión de expertos)

16. Ablación por catéter en pacientes con síncope y fibrilación auricular (opinión de expertos)

17. Indicación de desfibrilador en pacientes con fracción de eyección mayor de $35 \%$ y síncope

18. Indicación de desfibrilador en pacientes con síncope y cardiomiopatía hipertrófica de alto riesgo

19. Indicación de desfibrilador en pacientes con síncope y displasia arritmogénica

20. Evaluación psiquiátrica en pacientes con pseudo-síncope psicógeno

I $\quad \mathrm{IIb}$

IIb IIa

I IIa

IIb $\quad$ I

IIb IIa

PI: prueba de inclinación; HV: His-ventrículo

ción del ventrículo izquierdo (FEVI). Alto riesgo de muerte súbita.

\section{Unidad de síncope}

Las guías definen en detalle qué es una unidad de síncope, cómo diseñarla y su logística. También todos los procesos diagnósticos con numerosos flujogramas (cómo y cuándo realizar estudios diagnósticos), estructura, miembros, equipamiento, procedimientos.

Acceso y derivación a unidad de síncope: la derivación puede ser realizada por cualquier médico, de emergencia, de familia o de otros servicios, o incluso por el mismo paciente. Se recomienda un fácil acceso con lista de espera separada y visitas de seguimiento programadas. En particular, los pacientes de riesgo bajo/intermedio admitidos en el departamento de emergencia deberían beneficiarse de este tipo de logística de vía rápida a fin de reducir las tasas de ingresos, siendo dados de alta directamente desde la emergencia o después de una breve estadía en una unidad de observación.

La Sociedad Europea de Arritmias (EHRA) ha establecido diversos criterios de calidad de una unidad de síncope, que se resumen en la tabla 7. A pesar de 
Tabla 7. Unidad de síncope. Indicadores de calidad. Modificado de referencia 3.

1. La tasa absoluta de TLOC sin diagnóstico debe reducirse en un $20 \%$.

2. Menos del $20 \%$ de los pacientes con TLOC de riesgo bajo/intermedio deben ser admitidos al hospital.

3. Disminuir reingresos por síncope a $5 \%$.

4. Reducir los costos en un $20 \%$.

5. Al menos asistir 100 pacientes por año.

6. Especialista y equipo deben estar acreditados y concurrir regularmente a programas de actualización y congresos.

7. Tests:

a. $>95 \%$ ECG.

b. $>90 \%$ test de hipotensión ortostática, PI, registrador de eventos externo o implantable.

TLOC: pérdida de conocimiento transitoria;ECG: electrocardiograma; PI: prueba de inclinación.

las recomendaciones, desarrollar una unidad de síncope no es fácil, las barreras para establecerla incluyen falta de recursos y de personal capacitado. Por estos motivos no se han generalizado en la práctica.

\section{Comentarios finales}

Las nuevas guías dejan claro que se ha avanzado en el conocimiento y la comprensión del síncope, abordándolo como un evento complejo. Estimula el uso de nuevas tecnologías y herramientas visuales para su mejor diagnóstico.

En la práctica diaria existe una gran variación en el manejo de los pacientes con síncope y dificultad para adoptar las recomendaciones. Hay un vacío entre la evidencia científica disponible actualmente y lo que se hace en la práctica, demostrando incapacidad para difundir y aplicar estos conceptos en la clínica.

La ausencia de un enfoque sistemático genera mayores costos sanitarios, hospitalizaciones innecesarias, procedimientos diagnósticos incorrectos, prolongación de la estadía hospitalaria, menores tasas de diagnóstico, más errores diagnósticos y mayor tasa de recurrencias.
Por último, se reconoce que aún falta información y la necesidad de estudios clínicos en numerosos pacientes que evalúen el rendimiento diagnóstico y el cumplimiento de una estrategia sistemática generalizada.

Se recomiendan las siguientes líneas de investigación, estudios controlados aleatorizados que evalúen la eficacia de:

1) Tratamientos farmacológicos dirigidos a subgrupos específicos de síncope reflejo.

2) Uso de marcapasos para subgrupos específicos de síncope reflejo con formas cardioinhibitorias.

3) Tratamiento farmacológico del síncope mediado por hipotensión ortostática.

4) Implante de cardiodesfibrilador para subgrupos específicos de pacientes con síncope inexplicado con alto riesgo de muerte súbita cardíaca.

\section{Video}

En este video (http://www.suc.org.uy/revista/v33n3/ pdf/rcv33n3_vanerio-sincope-video.mp4) se muestran varias PI en pacientes con síncope reflejo que presentaron movimientos anormales. Las imágenes se explican por sí solas. Son muy diferentes a las crisis epilépticas y de muy breve duración, como clonias, sacudidas de cabeza o de brazos. En otros casos se pueden ver cambios en el tono con desviación cefálica o hiperextensión de todo el cuerpo.

\section{Bibliografía}

1. Brignole M, Moya A, de Lange FJ, Deharo JC, Elliott PM, Fanciulli A, et al. 2018 ESC Guidelines for the diagnosis and management of syncope. Eur Heart J. 2018;39 (21):1883-948. doi: 10.1093/ eurheartj/ehy037

2. Brignole M, Moya A, de Lange FJ, Deharo JC, Elliott PM, Fanciulli A, et al. Practical instructions for the 2018 ESC Guidelines for the diagnosis and management of syncope. Eur Heart J 2018; 39(21):e43-e80. doi:10.1093/eurheartj/ehy071

3. Kenny RA, Brignole M, Dan GA, Deharo JC, Van Dijk JG, Doherty C, et al. Syncope Unit: rationale and requirment - the European Heart Rhythm Association position statement endorsed by the Heart Rhythm Society. Europace 2015;17(9):1325-40. doi: $10.1093 /$ europace/euv115 